\title{
Application of a Fully Numerical Guidance to Mars Aerocapture
}

\author{
Daniel A. Matz* \\ NASA Johnson Space Center, 2101 NASA Parkway, Houston, TX r7058 \\ Ping $\mathrm{Lu}^{\dagger}$ \\ San Diego State University, San Diego, CA 92182 \\ Gavin F. Mendeck ${ }^{\ddagger}$ and Ronald R. Sostaric* \\ NASA Johnson Space Center, 2101 NASA Parkway, Houston, TX rro58
}

\begin{abstract}
An advanced guidance algorithm, Fully Numerical Predictor-corrector Aerocapture Guidance (FNPAG), has been developed to perform aerocapture maneuvers in an optimal manner. It is a model-based, numerical guidance that benefits from requiring few adjustments across a variety of different hypersonic vehicle lift-to-drag ratios, ballistic coefficients, and atmospheric entry conditions. In this paper, FNPAG is first applied to the Mars Rigid Vehicle (MRV) mid lift-to-drag ratio concept. Then the study is generalized to a design map of potential Mars aerocapture missions and vehicles, ranging from the scale and requirements of recent robotic to potential human and precursor missions. The design map results show the versatility of FNPAG and provide insight for the design of Mars aerocapture vehicles and atmospheric entry conditions to achieve desired performance.
\end{abstract}

\section{Nomenclature}

$\beta \quad$ Ballistic coefficient

$\gamma_{i} \quad$ Areocentric inertial atmospheric entry flight path angle

$\Delta V \quad$ Velocity change

$\sigma_{d} \quad$ Exit phase bank angle used when searching for switching time

dust $\tau \quad$ Measure of atmospheric dust optical depth

$C_{D} \quad$ Drag coefficient

$C_{L} \quad$ Lift coefficient

$L / D \quad$ Lift-to-drag ratio

$V_{i} \quad$ Inertial atmospheric entry velocity magnitude

FAST Flight Analysis and Simulation Tool

FNPAG Fully Numerical Predictor-corrector Aerocapture Guidance

MRV Mars Rigid Vehicle

MSL Mars Science Laboratory

\section{Introduction}

A new guidance algorithm, Fully Numerical Predictor-corrector Aerocapture Guidance (FNPAG), has been developed to fly optimal aerocapture maneuvers. ${ }^{1}$ FNPAG is a model-based, numerical guidance

\footnotetext{
*Aerospace Engineer, Flight Mechanics and Trajectory Design Branch, AIAA Senior Member.

${ }^{\dagger}$ Professor and Chair, Department of Aerospace Engineering, AIAA Fellow.

${ }^{\ddagger}$ Aerospace Engineer, Flight Mechanics and Trajectory Design Branch.
} 
and is thus adaptable to a wide range of vehicle designs and flight conditions without the need for intricate tuning or reference trajectory generation.

The original development of FNPAG considered aerocapture at Earth. In this paper, FNPAG is now applied to aerocapture at Mars. First, FNPAG is used for the design of the Mars Rigid Vehicle (MRV), a mid lift-to-drag ratio concept for human scale Mars missions. ${ }^{2}$ Then, in order to further test the performance of FNPAG, it is applied to a design map of potential Mars aerocapture missions and vehicles.

Design maps are sets of data that span key vehicle, trajectory, and mission parameters. They are effective at providing insight into the design space, allowing the engineering team to identify important trends and valuable trades. Design maps are thus useful for conceptual design studies, where careful selection of vehicle and mission parameters can make a concept feasible. They are also useful for mission design, where vehicle parameters are fixed but trajectory and mission parameters can be adjusted to increase performance.

Design maps have been used for many decades. An early example are pork chop plots, which show the $\Delta V$ requirements for interplanetary transfers over a scan of departure dates and transfer times. ${ }^{3}$ More recently, design maps were used to select settings for the Mars Science Laboratory (MSL) entry guidance. ${ }^{4}$

The aerocapture design map developed in this paper encompasses a design space that includes ballistic coefficient, lift-to-drag ratio, and atmospheric entry conditions spanning from recent robotic missions to potential future human and precursor missions. The design map showcases the adaptability of the FNPAG algorithm. It also identifies several areas of improvement to guide future FNPAG development. Additionally, the design map provides insight into the trends that drove the design of the MRV aerocapture maneuver. It is hoped that the design map will prove to be a useful resource for future Mars aerocapture conceptual design studies, by providing performance estimates early on in the vehicle design process and good starting points for detailed Monte Carlo studies.

\section{Review of FNPAG}

The longitudinal channel of FNPAG determines the magnitude of the bank angle command, which is the trajectory control for aerocapture. Optimal control theory shows that the bank angle magnitude profile that minimizes the post-aerocapture in-plane orbit correction $\Delta V$ has a "bang-bang" structure: the vehicle should first fly at the smallest allowable bank angle magnitude and then switch to the largest allowable bank angle magnitude at an appropriate time. ${ }^{1}$ FNPAG was thus implemented as a two-phase algorithm. In Phase 1, the predicted bank angle profile is parameterized by a stair function as shown in Figure 1, where $\sigma_{0}$ and $\sigma_{d}$ are user-specified constants that are chosen to balance the $\Delta V$ performance and trajectory control margins. The algorithm searches for the switching time, $t_{s}$, to target the specified apoapsis or minimize the total post-aerocapture in-plane $\Delta V$. The solution process to find $t_{s}$ is repeated in each guidance cycle to realize closed-loop guidance. Once the trajectory passes $t_{s}$, Phase 2 of the algorithm begins. The algorithm searches for a constant bank angle profile to target the specified apoapsis or minimize the total post-aerocapture inplane $\Delta V$. As before, this is done at each guidance cycle. In this way, FNPAG maintains closed-loop guidance throughout the aerocapture maneuver. For a more detailed explanation of FNPAG, see Ref. 1.

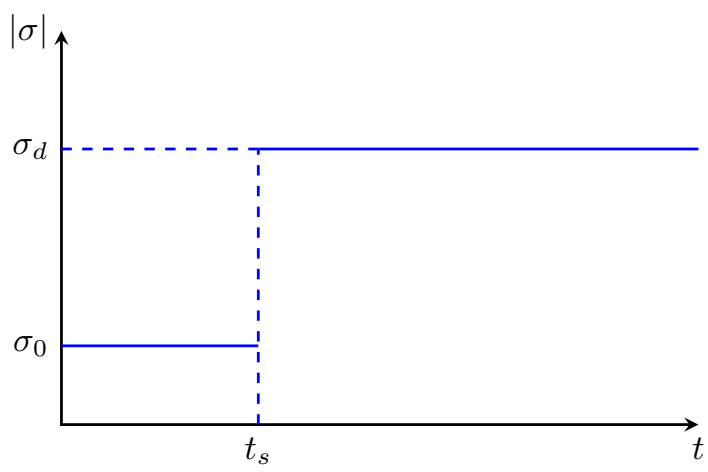

Figure 1: Bank angle magnitude profile in phase 1 of FNPAG

In the lateral channel FNPAG originally used the Apollo-heritage lateral error deadbands for the determination of the bank angle sign. Recently, a new approach to lateral logic was developed for numerical 
guidance algorithms. ${ }^{5}$ Two additional trajectory propagations are performed to predict the bank left and bank right crossranges, and the resulting crossrange values are used to command bank reversals following a geometric reduction of the final crossrange error. This approach has the unique property of allowing the number of bank reversals to be specified a priori. This approach has been adopted for use in FNPAG.

\section{Mars Rigid Vehicle}

The MRV is a proposed concept for a human scale Mars entry vehicle, capable of delivering 20 metric tons of payload to the surface of Mars. ${ }^{2}$ It is a rigid, enclosed, elongated lifting body with an $L / D$ higher than that of a typical entry capsule but lower than that of a typical winged entry vehicle. A rendering of the MRV is shown in Figure 2. For a more detailed description of the MRV concept, see Ref. 2. This section presents an atmospheric entry flight path angle corridor assessment, nominal trajectory, and Monte Carlo assessment of the MRV aerocapture into a highly elliptical 1 sol orbit.

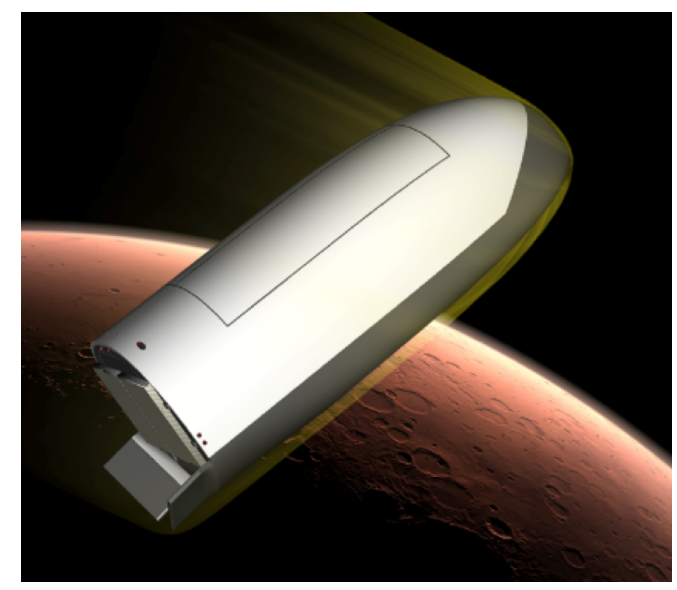

Figure 2: The Mars Rigid Vehicle is a rigid lifting body

\section{A. Simulation Configuration and Assumptions}

The MRV aerocapture trajectory was simulated using the Flight Analysis and Simulation Tool (FAST). FAST was configured to simulate a 3 degree-of-freedom vehicle. The MRV utilizes a split body flap to maintain a trim angle of attack of $55 \mathrm{deg}$. The aerodynamic model used a constant lift coefficient of 1.36 and a constant drag coefficient of 2.51, resulting in a lift-to-drag ratio of 0.54 . The mass at atmospheric entry was 60 metric tons and the aerodynamic reference area was $65 \mathrm{~m}^{2}$, resulting in a ballistic coefficient of $368 \mathrm{~kg} / \mathrm{m}^{2}$. The initial condition was a radius of $3522 \mathrm{~km}$, planet-fixed declination of $81.6 \mathrm{deg}$, inertial velocity magnitude of $6.2 \mathrm{~km} / \mathrm{s}$, and areocentric inertial azimuth of $180 \mathrm{deg}$.

Mars GRAM $2010^{6}$ was used to model the atmosphere, with Mars GRAM called as a subroutine as the trajectory was integrated. For the corridor width assessment and the nominal trajectory, no winds were modeled. For the Monte Carlo analysis, Mars GRAM winds were used.

FNPAG was provided with undispersed vehicle mass, aerodynamic reference area, lift coefficient, and drag coefficient and a nominal atmospheric density profile. FNPAG was configured to target a post-aerocapture altitude of apoapsis of $33,793 \mathrm{~km}$ and an altitude of periapsis of $250 \mathrm{~km}$. For the lateral channel, FNPAG was configured to target an inclination of $90 \mathrm{deg}$. FNPAG was configured to use its Mode 1, which performs the optimal aerocapture maneuver while targeting the desired altitude of apoapsis. The $\sigma_{d}$ parameter, which controls the amount of bank angle margin during the exit phase, was set to 90 deg. FNPAG was called at $1 \mathrm{~Hz}$, and its bank angle command was then passed to a model which emulated the control and effector response using a $\mathrm{PD}$ controller with a maximum bank angle rate of $15 \mathrm{deg} / \mathrm{s}$ and a maximum bank angle acceleration of $5 \mathrm{deg} / \mathrm{s}^{2}$. 


\section{B. Corridor Width Assessment}

An undispersed, open loop, full lift up case was run iteratively using a bisection search to determine the atmospheric entry flight path angle of the trajectory that achieves the targeted altitude of apoapsis at atmospheric exit. This defines the steepest possible flight path angle. The same was repeated for an undispersed, open loop, full lift down case, which then defines the shallowest possible flight path angle. For the MRV, the inertial steep corridor boundary was identified to be -12.9 deg and the inertial shallow corridor boundary identified to be $-11.0 \mathrm{deg}$. Both flight path angles are defined at the MRV initial condition.

\section{Nominal Trajectory}

For the MRV, the peak heat rate during aerocapture exceeds that during entry and thus drives the thermal protection system design. In order to minimize the peak heat rate, the nominal atmospheric entry flight path angle was selected to be as shallow as possible. The corridor analysis above is for undispersed trajectories, so the nominal flight path angle must be steeper. Beginning with a small offset from the shallow boundary, a Monte Carlo was run iteratively, adjusting the flight path angle to be as shallow as possible while achieving 3 -sigma success. This process resulted in the selection of a nominal atmospheric entry flight path angle of $-11.3 \mathrm{deg}$. The nominal trajectory and nominal bank angle profile is shown in Figure 3. The trajectory begins at $6.2 \mathrm{~km} / \mathrm{s}$, on the right of the plot, then dips into the atmosphere to bleed off energy, and finally exits the atmosphere on the left of the plot at roughly $4.75 \mathrm{~km} / \mathrm{s}$. The bank angle is plotted as a function of time. Before entering the sensible atmosphere, FNPAG commands a small bank angle magnitude, near the full lift up bank angle of 0 deg. When the sensed acceleration magnitude exceeds 0.2 Mars g, at $85 \mathrm{~s}$, Phase 1 of the guidance begins. This phase would normally continue to orient the lift direction upward by commanding a small non-zero bank angle. The command is not exactly zero so that some of the lift can be used for lateral control. For this case, however, the trajectory is so shallow that Phase 1 immediately ends and FNPAG proceeds to Phase 2. FNPAG commands a bank reversal to orient the lift vector downward, where full lift down would be a bank angle of $180 \mathrm{deg}$. As the MRV leaves the atmosphere, it loses control authority while still trying to reduce the remaining apoapsis error, causing the bank angle to saturate nearly full lift up. After about $300 \mathrm{~s}$, the MRV has left the sensible atmosphere.
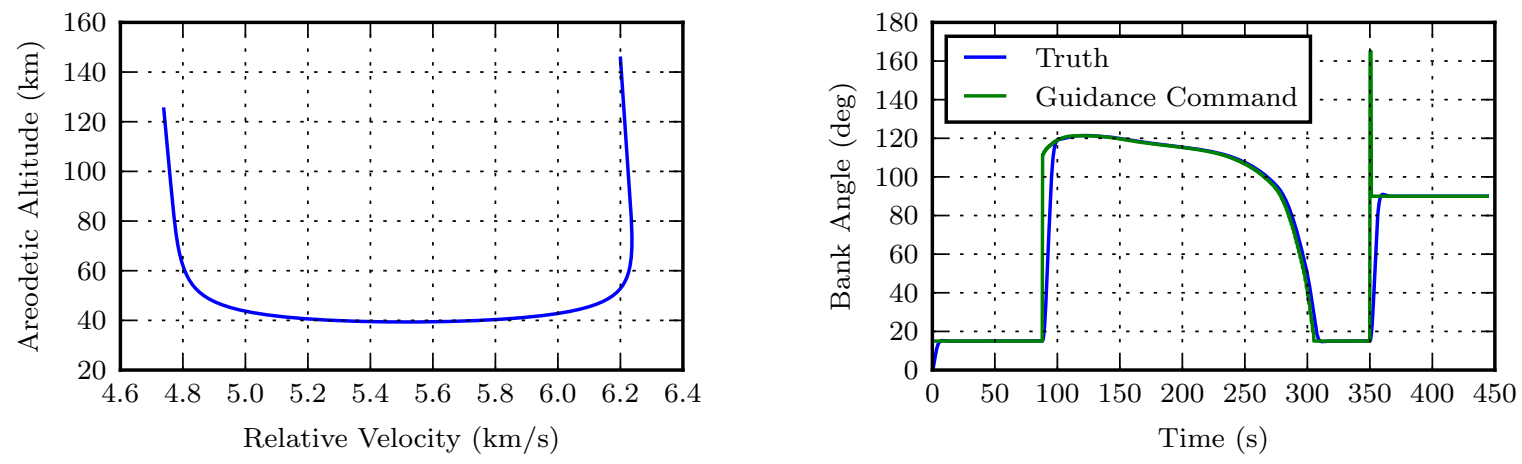

Figure 3: The nominal MRV aerocapture trajectory

\section{Monte Carlo}

A Monte Carlo analysis was used to determine the peak heat rate for a $1 \mathrm{~m}$ nose radius and the $\Delta V$ budget for the post-aerocapture in-plane orbital corrections. The atmospheric entry flight path angle, dust $\tau$, lift coefficient, and drag coefficient were all dispersed uniformly, and the dispersion levels are shown in Table 1. The dust $\tau$ parameter is a measure of the opacity of the atmosphere and influences the atmospheric density profile. The Mars GRAM random seed for the atmospheric density profile was also dispersed.

As discussed above, the nominal flight path angle of $-11.3 \mathrm{deg}$ is as shallow as possible. The Monte Carlo results in one of the 3000 cases skipping out, that is, exiting the atmosphere on a hyperbolic orbit. A single skip out was considered acceptable given the conservative dispersion assumptions at this early phase of the 
Table 1: Dispersions

\begin{tabular}{lc}
\hline Variable & Dispersion \\
\hline$\gamma_{i}$ & $\pm 0.2 \mathrm{deg}$ \\
dust $\tau$ & 0.1 to 0.9 \\
$C_{L}$ & $\pm 10 \%$ \\
$C_{D}$ & $\pm 10 \%$ \\
\hline
\end{tabular}

design. A histogram of the post-aerocapture in-plane orbital correction $\Delta V$ for insertion into the $250 \mathrm{~km}$ by $33,793 \mathrm{~km}$ target orbit is shown in Figure 4 . A value of $40 \mathrm{~m} / \mathrm{s}$ was budgeted for the MRV design.

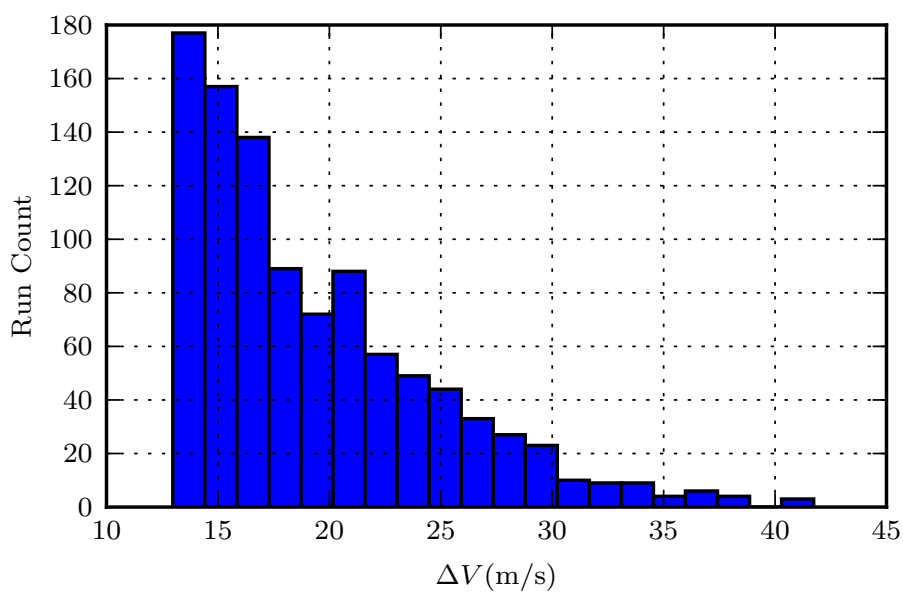

Figure 4: The MRV post-aerocapture in-plane orbital correction $\Delta V$ from a 3000 case Monte Carlo

\section{Design Map}

The design space is a parametric sweep of ballistic coefficient, lift-to-drag ratio, inertial atmospheric entry velocity magnitude, and areocentric inertial atmospheric entry flight path angle. The bounds were selected to encompass both recent robotic missions and future human and precursor missions. The design space is shown in Table 2 and is compared to flown and proposed vehicles in Figure 5.

Table 2: The design space includes vehicle parameters and atmospheric entry conditions

\begin{tabular}{ll}
\hline Variable & Values \\
\hline$\beta$ & $100,150, \ldots, 500 \mathrm{~kg} / \mathrm{m}^{2}$ \\
$L / D$ & $0.18,0.24,0.3,0.35, \ldots, 0.6$ \\
$V_{i}$ & $5.4,5.6, \ldots, 7.4 \mathrm{~km} / \mathrm{s}$ \\
$\gamma_{i}$ & $-14.8,-14.7, \ldots,-7.9 \mathrm{deg}$ \\
\hline
\end{tabular}

This design map represents dispersed performance. The same dispersion levels are used for the design map as were used for the MRV Monte Carlo analysis and are shown in Table 1. Again, all dispersions use a uniform distribution.

The design space contains 62,370 design points. If a 3000 case Monte Carlo were run at each design point, a total of 187 million cases would need to be simulated. This was clearly not feasible with current 


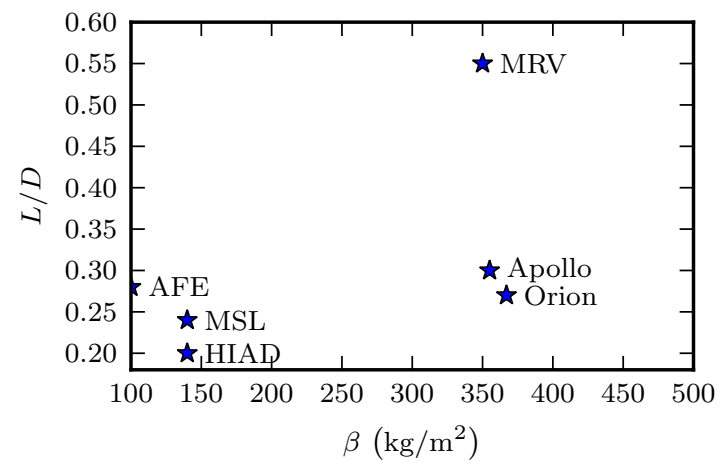

Figure 5: The design space encompasses both flown and proposed vehicles

computing resources. Instead, a set of stress cases were selected which reasonably represent the dispersed performance across the entire design space.

\section{A. Simulation Configuration and Assumptions}

FAST was again configured to simulate a 3 degree-of-freedom vehicle. The aerodynamic model used a constant lift and drag coefficient. The combination of vehicle mass, aerodynamic reference area, lift coefficient, and drag coefficient were selected to achieve the desired ballistic coefficient and lift-to-drag ratio.

The initial condition was at an areodetic altitude of $125 \mathrm{~km}$, longitude of $0 \mathrm{deg}$, areodetic latitude of $0 \mathrm{deg}$, and an areocentric inertial azimuth of $80 \mathrm{deg}$.

For the Monte Carlo cases, FAST was configured to call Mars GRAM as a subroutine as the trajectory was integrated. For the stress cases, several dispersed density profiles were generated using the stand alone Mars GRAM executable and then linearly interpolated by FAST, so that the stress cases at various design points used a consistent atmosphere. No winds were modeled.

The time and date were chosen to yield a local solar time of 12:00, when the atmospheric entry location is facing the sun, and thus also facing in the general direction of Earth. This configuration is preferred to allow communication with the spacecraft.

The design map was generated for the same reference mission that was used for the MRV design, that is an aerocapture into a 1-sol elliptical orbit around Mars with an altitude of apoapsis of 33,793 km and altitude of periapsis of $250 \mathrm{~km}$, which has been used for Mars studies since Design Reference Architecture (DRA) 5.0..$^{7,8}$

FNPAG was called at $1 \mathrm{~Hz}$. It was configured to use its Mode 1, which performs the optimal aerocapture maneuver while targeting the desired altitude of apoapsis. The $\sigma_{d}$ parameter, which controls the amount of bank angle margin during the exit phase, was set to $90 \mathrm{deg}$, which has proven to be a reasonable value for a variety of cases. The FNPAG target inclination was set to $10 \mathrm{deg}$. The FNPAG bank command was flown directly, effectively resulting in an infinite bank angle rate.

FNPAG was provided the undispersed lift coefficient, drag coefficient, and mass. Though dust $\tau$ was being dispersed, it was assumed to be known by the guidance to a certain degree. Three nominal atmosphere profiles were generated for dust $\tau$ values of $0.1,0.5$, and 0.9 , and the one that most closely matched the dispersed dust $\tau$ was used within FNPAG when integrating the predicted trajectory. Otherwise, the same FNPAG configuration was used across the entire design space.

\section{B. Stress Cases}

The stress cases were selected by comparing candidate stress cases to a Monte Carlo at sample design points across the design space. The sample design points are shown in Table 3.

The candidate stress cases were defined by evenly discretizing each dispersion into five dispersion levels. Drawing repeatedly from the five discretized dust $\tau$ values, Mars GRAM 2010 was used to generate 80 dispersed atmospheres. Taking the combination of the remaining discretized dispersions and the 80 atmospheres yielded 10,000 candidate stress cases. 
Table 3: A set of sample design points were used for selecting stress cases

\begin{tabular}{cccc}
\hline $\begin{array}{c}\beta \\
\left(\mathrm{kg} / \mathrm{m}^{2}\right)\end{array}$ & $L / D$ & $\begin{array}{c}V_{i} \\
(\mathrm{~km} / \mathrm{s})\end{array}$ & $\begin{array}{c}\gamma_{i} \\
(\mathrm{deg})\end{array}$ \\
\hline 100 & 0.18 & 6.4 & -10.09 \\
100 & 0.4 & 6.4 & -10.27 \\
100 & 0.6 & 6.4 & -10.55 \\
300 & 0.18 & 6.4 & -10.68 \\
300 & 0.4 & 6.4 & -10.87 \\
300 & 0.6 & 6.4 & -11.17 \\
500 & 0.18 & 6.4 & -10.95 \\
500 & 0.4 & 6.4 & -11.15 \\
500 & 0.6 & 6.4 & -11.43 \\
\hline
\end{tabular}

The candidate stress cases and a 3000 case Monte Carlo were then run at each sample design point. The results were compared in terms of the following performance metrics: post-aerocapture in-plane orbital correction $\Delta V$, peak sensed acceleration magnitude, peak heat rate, heat load, and minimum altitude. The selected set of stress cases was required to reasonably represent the Monte Carlo at each design point. Specifically, at least one stress case had to be within $\pm 10 \%$ of the $99.7^{\text {th }}$ percentile of the peak sensed acceleration, $\pm 5 \%$ of the $99.7^{\text {th }}$ percentile of the peak heat rate and heat load, $\pm 5 \%$ of the $0.3^{\text {rd }}$ percentile of the minimum altitude, and between the $99^{\text {th }}$ and $99.7^{\text {th }}$ percentiles of the post-aerocapture in-plane orbital correction $\Delta V$ from the Monte Carlo. If a stress case was outside of the required tolerance and was overconservative for any metric at any sample design point, it was eliminated from consideration. If a stress case was outside of the required tolerance and was under-conservative, it was not disqualified, because it may have been a good stress case for a different metric or design point.

This process is illustrated in Figure 6, where five stress cases are analyzed at two design points for a single metric. The dashed horizontal lines show the bounds from the Monte Carlo at each design point. Stress case 1 is over-conservative at design point 1, and is therefore eliminated, even though it is a good stress case at design point 2. Stress case 2 is good at both design points. Stress case 3 is not stressing enough at either design point. Stress case 4 is not stressing enough at design point 1 , but is a good case for design point 2 . And stress case 5 is a good case for design point 1 , but not stressing enough at design point 2 . Given these cases, only stress cases 2,4 , and 5 can be considered. The best choice would be to use stress case 2 only, as it represents the metric at both design points. But using stress cases 4 and 5 as a set would also be a valid choice.
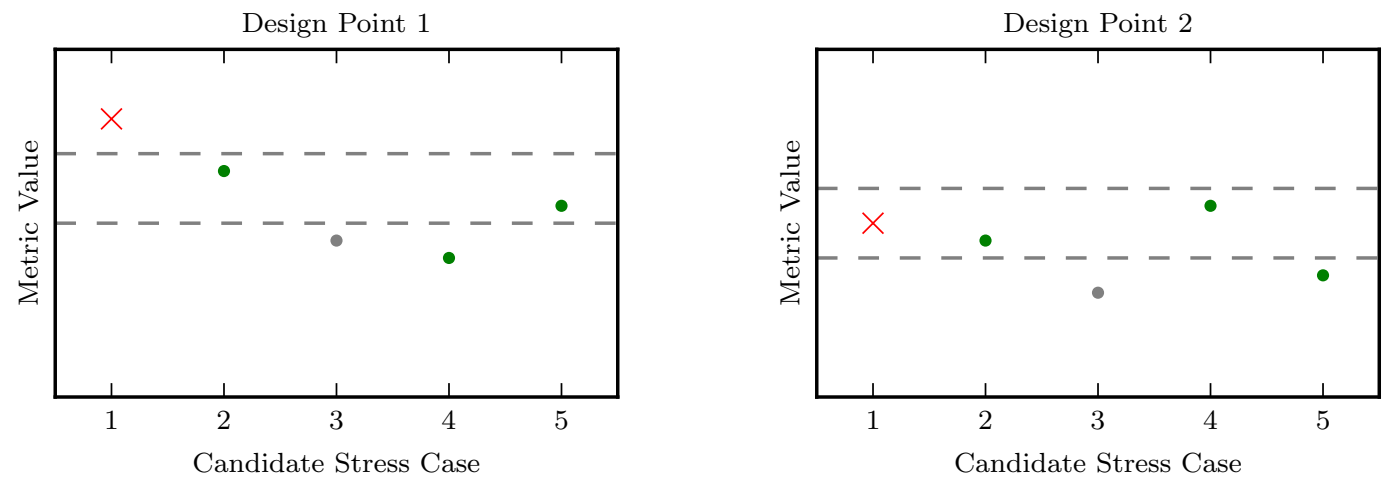

Figure 6: Stress cases were selected by comparing candidate stress cases to the Monte Carlo output 
This process was applied across all 45 design point and metric combinations, and a set of 8 stress cases were identified. They are shown in Table 4 . The dispersed atmospheres used by the selected stress cases are shown in Figure 7.

Table 4: A set of stress cases were identified to represent the Monte Carlo results

\begin{tabular}{ccccc}
\hline $\begin{array}{c}\Delta \gamma_{i} \\
(\mathrm{deg})\end{array}$ & $\begin{array}{c}\Delta C_{D} \\
(\%)\end{array}$ & $\begin{array}{c}\Delta C_{L} \\
(\%)\end{array}$ & Dust $\tau$ & Atmosphere Index \\
\hline-0.1 & -10 & -10 & 0.3 & 11 \\
-0.2 & -10 & -5 & 0.7 & 33 \\
+0.1 & -10 & +10 & 0.3 & 46 \\
-0.2 & +5 & -10 & 0.3 & 11 \\
-0.2 & -10 & +5 & 0.1 & 45 \\
+0.2 & -10 & +10 & 0.1 & 75 \\
+0.2 & -10 & -10 & 0.5 & 67 \\
-0.2 & +10 & -10 & 0.9 & 74 \\
\hline
\end{tabular}
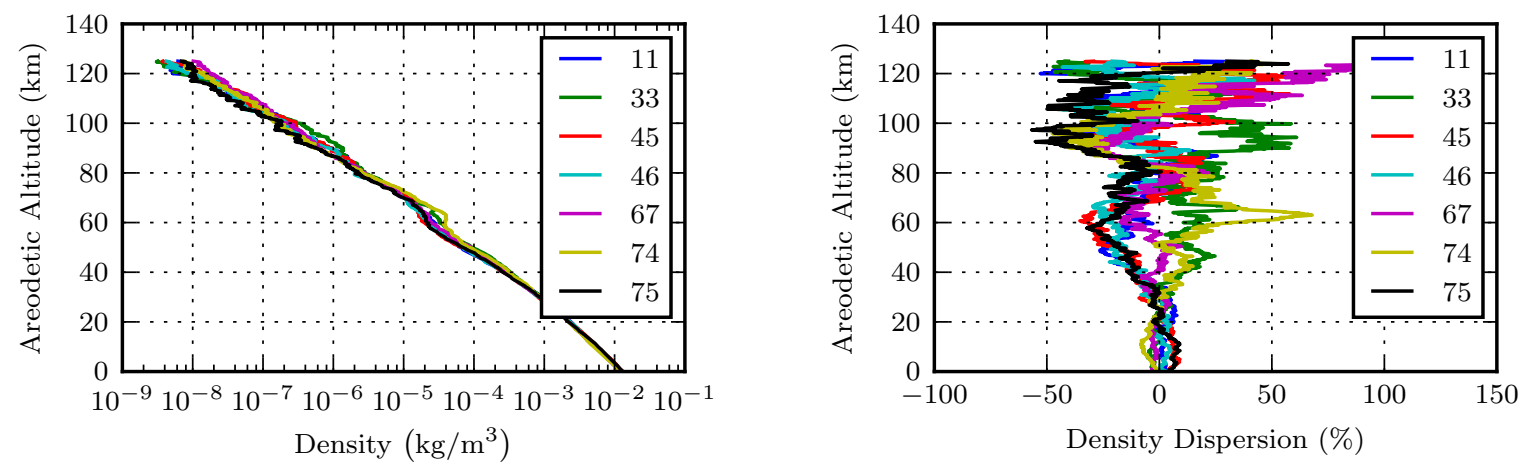

Figure 7: Dispersed atmospheres used by the selected stress cases

\section{Results}

This section describes several ways in which the design map can be applied to vehicle and mission design, using the MRV as an example. The design point closest to the MRV parameters is used for comparison. The MRV ballistic coefficient is $368 \mathrm{~kg} / \mathrm{m}^{2}$, so the design map value of $350 \mathrm{~kg} / \mathrm{m}^{2}$ is used. The MRV $L / D$ is 0.54 , and the nearest design map value is 0.55 . The MRV initial condition was specified at a higher altitude and a different azimuth, so the relative velocities at $125 \mathrm{~km}$ were matched by using the design map atmospheric entry inertial velocity of $6.4 \mathrm{~km} / \mathrm{s}$. The flight path angles discussed above for the MRV were also specified at the higher altitude initial condition, and they will be converted to the corresponding values at $125 \mathrm{~km}$ as needed throughout this section.

The first vehicle design application is to analyze the dispersed flight path angle corridor as a function of $\beta$ and $L / D$. This is shown for a fixed inertial velocity of $6.4 \mathrm{~km} / \mathrm{s}$ in Figure 8 . For such a highly elliptical target orbit, the shallow side of the corridor is essentially defined by skipping out. For the steep side, cases have substantial room to miss the target apoapsis without actually capturing and entering. However, the $\Delta V$ becomes prohibitively high. So instead the steep boundary is defined by the $\Delta V$ exceeding $100 \mathrm{~m} / \mathrm{s}$. The shallow and steep boundaries depend on both $L / D$ and $\beta$, but the corridor width depends almost entirely on $L / D$.

In terms of inertial flight path angles at $125 \mathrm{~km}$, the undispersed MRV corridor is from $-11.8 \mathrm{deg}$ to $-9.7 \mathrm{deg}$. Because the design map corridor includes the effects of dispersions, the flight path angles at the 
shallow boundary are more negative with a value of -10.3 . The steep boundary compares rather well. The grayed-out regions show where there is no feasible corridor.

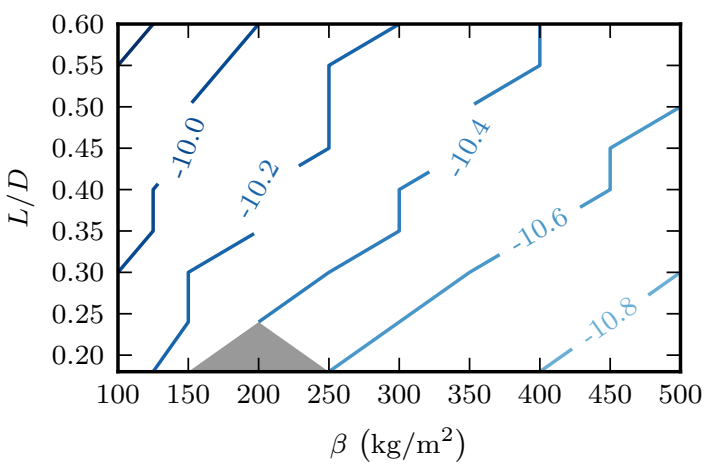

(a) Shallowest flight path angle (deg)

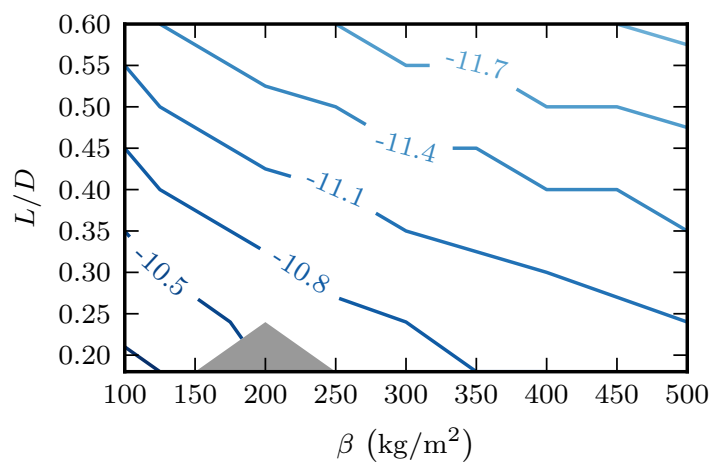

(b) Steepest flight path angle (deg)

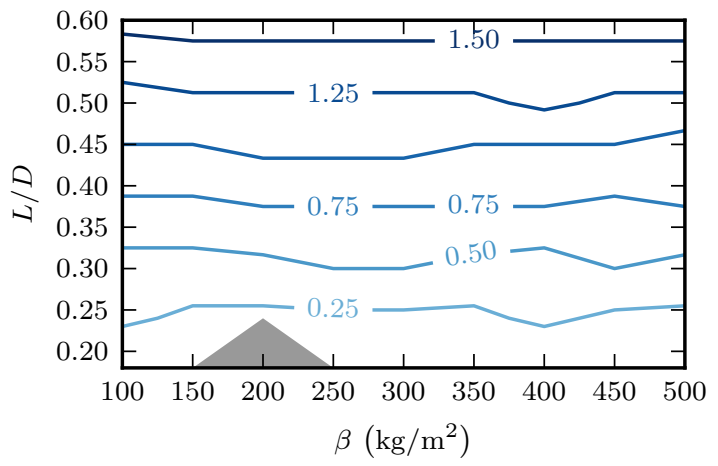

(c) Corridor width (deg)

Figure 8: Flight path angle corridor as a function of $\beta$ and $L / D$ for $V_{i}=6.4 \mathrm{~km} / \mathrm{s}$

Another vehicle design application is to allow the flight path angle to be selected to minimize the $\Delta V$ at each combination of $\beta$ and $L / D$. The resulting metrics are shown in Figure 9, again for a fixed inertial velocity of $6.4 \mathrm{~km} / \mathrm{s}$. For most of the combinations with $L / D$ of 0.3 or higher, FNPAG is able to perform the aerocapture while requiring a post-aerocapture in-plane $\Delta V$ less than $30 \mathrm{~m} / \mathrm{s}$. As the $L / D$ approaches the lower end of the design space the $\Delta V$ required increases sharply.

By selecting the flight path angle to minimize the in-plane $\Delta V$, the trajectories with higher $L / D$ make use of the vehicle's lifting capability by entering the atmosphere at steeper flight path angles, experiencing slightly higher peak accelerations. This effect is not strong enough to affect the peak heat rate and heat load, which are mostly influenced by ballistic coefficient.

Because the peak heat rate during aerocapture was a driving metric for the MRV, the most shallow atmospheric entry flight path angle possible was selected. The design map can also be used to study the performance at the shallow flight path angle corridor boundary, as shown in Figure 10. Compared to the previous set, the $\Delta V$ values are higher, though they generally show the same trend, increasing as $L / D$ approaches the lower bound. There is also some noise in the $\Delta V$ values, which is a result of the resolution of the flight path angle, such that some design points are much nearer the shallow boundary than others. In the previous set a higher $L / D$ resulted in higher peak acceleration, but when the flight path angle is chosen at the shallow corridor, a higher $L / D$ allows the vehicle to enter the atmosphere with a shallower flight path angle, go less deep into the atmosphere, and thus result in a lower peak acceleration. The shallower trajectories also result in lower heat rates, as compared to the previous set. The heat rate and heat load are mostly a function of the ballistic coefficient, though for higher ballistic coefficients a higher $L / D$ does enable a shallower trajectory and lower heat rates. 


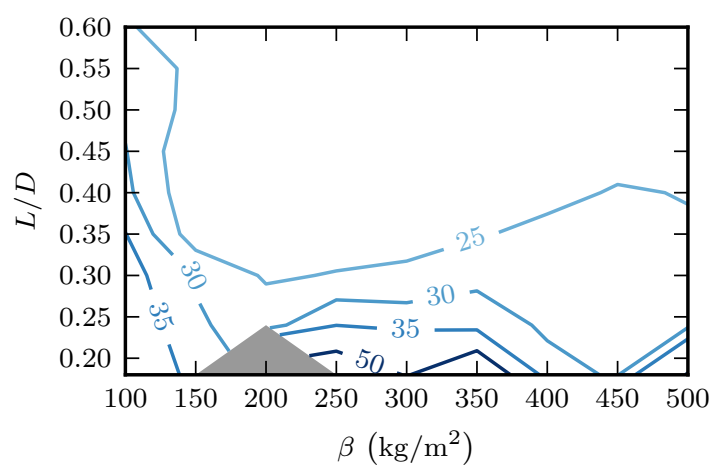

(a) In-plane $\Delta V(\mathrm{~m} / \mathrm{s})$

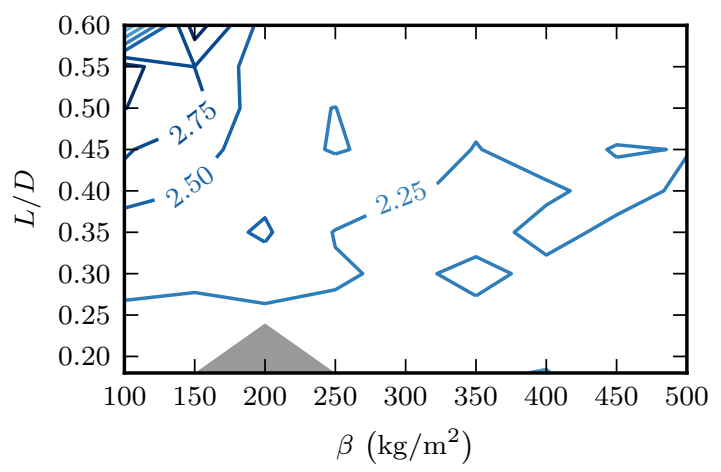

(c) Peak sensed acceleration magnitude (Earth g)

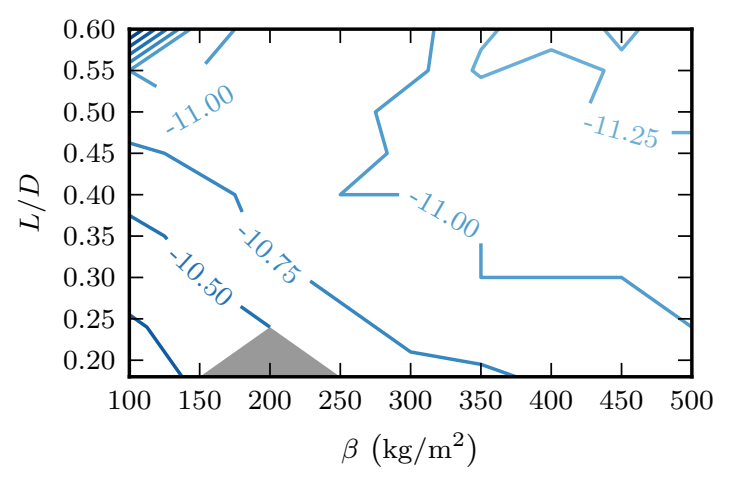

(b) Atmospheric entry flight path angle (deg)

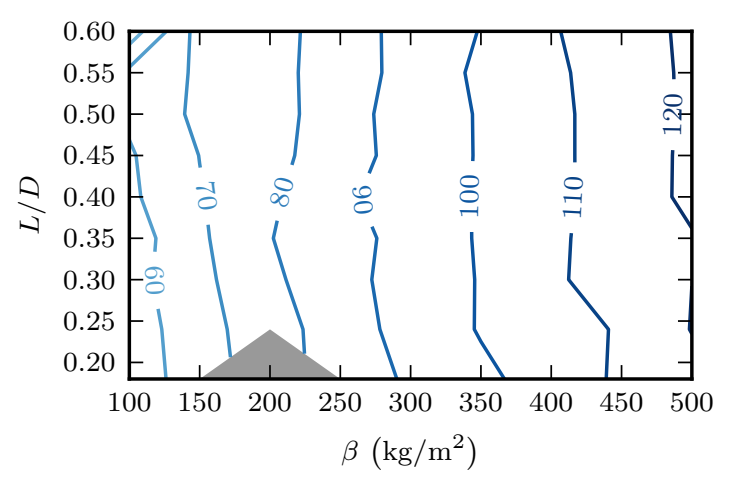

(d) Peak heat rate $\left(\mathrm{W} / \mathrm{cm}^{2}\right)$

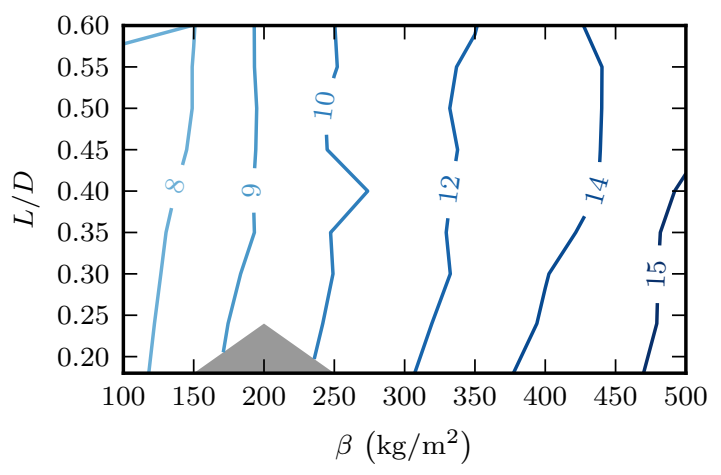

(e) Heat load $\left(\mathrm{kJ} / \mathrm{cm}^{2}\right)$

Figure 9: Select $\gamma_{i}$ where in-plane $\Delta V$ is minimized for $V_{i}=6.4 \mathrm{~km} / \mathrm{s}$ 


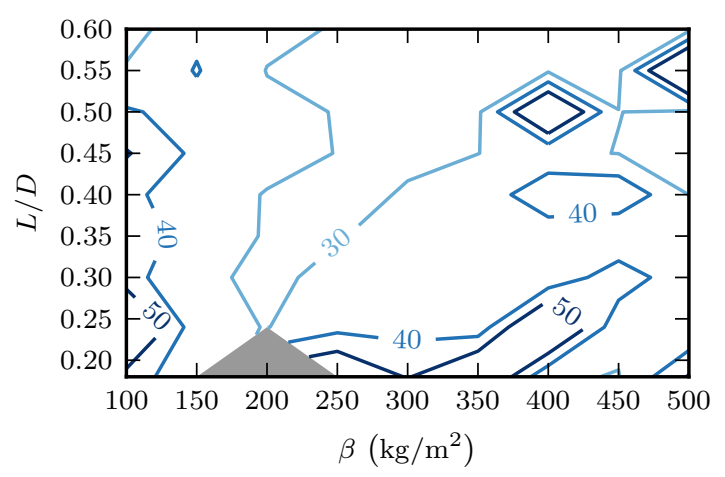

(a) In-plane $\Delta V(\mathrm{~m} / \mathrm{s})$

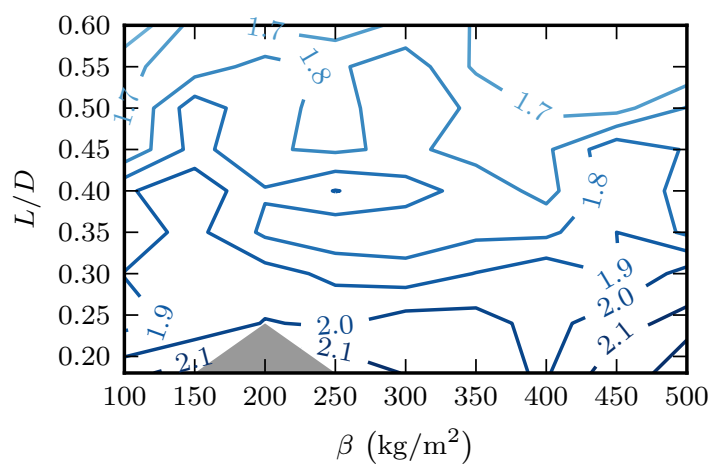

(c) Peak sensed acceleration magnitude (Earth g)

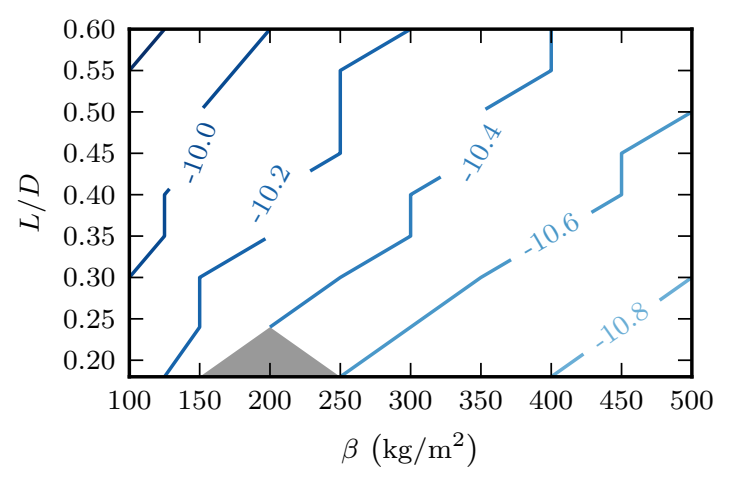

(b) Atmospheric entry flight path angle (deg)

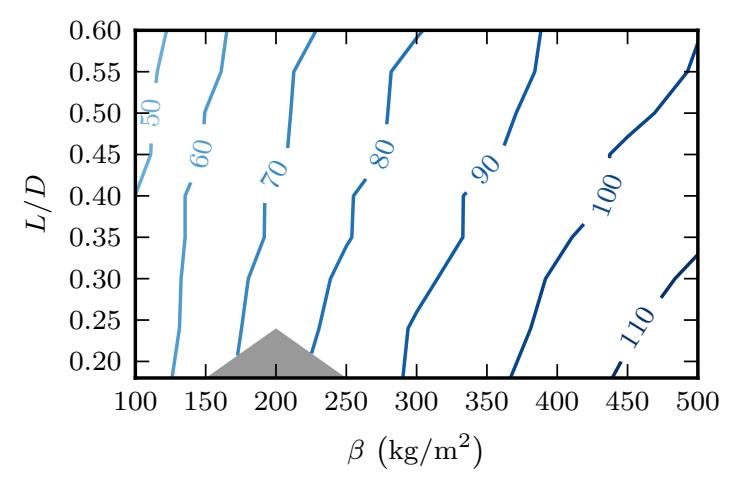

(d) Peak heat rate $\left(\mathrm{W} / \mathrm{cm}^{2}\right)$

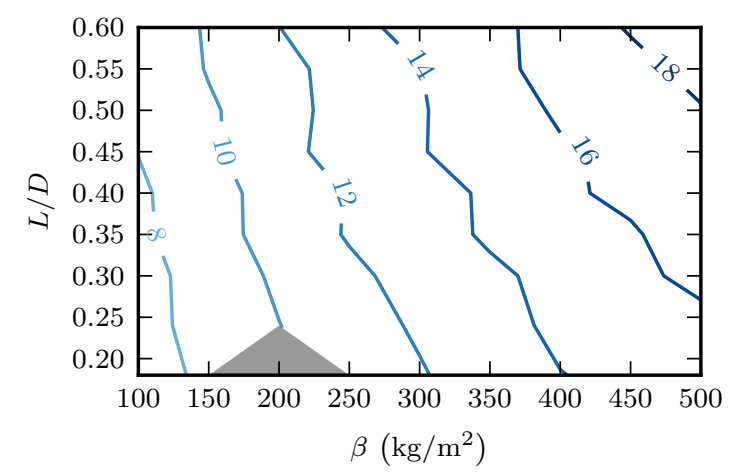

(e) Heat Load $\left(\mathrm{kJ} / \mathrm{cm}^{2}\right)$

Figure 10: Select $\gamma_{i}$ at the shallow boundary for $V_{i}=6.4 \mathrm{~km} / \mathrm{s}$ 
Another application of the design map is to look at the sensitivity of a driving metric to the vehicle design parameters. In the case of the MRV, the driving metric is heat rate. Figure 11 shows how heat rate changes as a function of the vehicle and atmospheric entry design parameters while holding the other parameters fixed. The grayed-out regions are outside of the flight path angle corridor.

The design map can also be used as the design becomes better defined. Consider the MRV case, and assume that the ballistic coefficient has been fixed. Figure 12 shows the in-plane $\Delta V$ as a function of $L / D$ and $\gamma_{i}$. The grayed-out regions are outside of the flight path angle corridor, showing the strong influence of $L / D$ on the flight path angle corridor width. The corridor width and $\Delta V$ can then inform the selection of $L / D$.

Once the $L / D$ is also selected, the design map can still be used to fine tune the selection of the atmospheric entry flight path angle. Figure 13 shows the performance metrics as functions of $\gamma_{i}$. Recall that the MRV flight path angle was selected as shallow as possible to reduce the heat rate. These trends support that decision. The design map shows the shallowest possible flight path angle is $-10.3 \mathrm{deg}$. The nominal MRV initial condition resulted in a flight path angle of $-10.1 \mathrm{deg}$ at $125 \mathrm{~km}$. Some of this discrepancy can be explained by the fact that the design map does not allow any skip out trajectories, while the MRV design did allow one. Also recall that the design point's $\beta$ and $L / D$ don't match the MRV perfectly. The differences in the initial conditions may also have some effect, including the effects of the initial latitude on the atmospheric density.

The shallow boundary design point can be compared to the MRV Monte Carlo. The $99^{\text {th }}$ percentile $\Delta V$ from the MRV Monte Carlo was $37.2 \mathrm{~m} / \mathrm{s}$, while the design map gives a value of $27.1 \mathrm{~m} / \mathrm{s}$. The $99.7^{\text {th }}$ percentile peak sensed acceleration magnitude from the MRV Monte Carlo was 1.84 Earthg, while the design map gives a value of 1.67 Earthg at the shallow boundary. The $99.7^{\text {th }}$ percentile peak heat rate from the MRV Monte Carlo was $91.7 \mathrm{~W} / \mathrm{cm}^{2}$, and the design map gives a value of $86.5 \mathrm{~W} / \mathrm{cm}^{2}$. The $99.7^{\text {th }}$ percentile heat load from the MRV Monte Carlo was $15.0 \mathrm{~kJ} / \mathrm{cm}^{2}$, while the design map gives a value of $15.6 \mathrm{~kJ} / \mathrm{cm}^{2}$. Lastly, the $0.3^{\text {rd }}$ percentile minimum altitude from the MRV Monte Carlo was $36.6 \mathrm{~km}$, and the design map gives a value of $38.7 \mathrm{~km}$.

These values compare moderately well. Some of the discrepancy can be attributed to not matching the MRV $\beta, L / D$, and $V_{i}$ perfectly with the design map. In addition, the shallow boundary $\gamma_{i}$ from the design map is slightly steeper than the MRV $\gamma_{i}$. Other aspects of the MRV initial condition do not match the design map, either, such as azimuth and latitude. Lastly, the MRV study included bank angle rate and acceleration limits, while the design map does not.

\section{Observations and Future Work}

The design map was originally developed using the same bank angle rate and acceleration limits that were used for the MRV Monte Carlo. Because the performance at a design point depended on such a small number of cases, a poorly timed bank reversal could greatly reduce the performance of a single case and then drive the output at that design point. This tended to confuse the trends. FNPAG does not currently limit when a bank reversal can be performed, and it is expected that improvements will be made to the algorithm so that finite bank reversals can be included in future design map efforts.

The entry version of FNPAG, called Fully Numerical Predictor-corrector Entry Guidance (FNPEG), includes heat rate limiting logic. Since heat rate is a driving metric for the MRV design, it may be worthwhile to consider implementing a similar feature into FNPAG.

While FNPAG waits until the vehicle enters the sensible atmosphere before beginning with Phase 1, it continues to command the bank angle long after it has exited the sensible atmosphere. This was not an issue for these studies, but it would be helpful to update FNPAG to detect when it exits the sensible atmosphere. The acceleration threshold could even be adjusted to trade orbit insertion accuracy against attitude control propellant usage.

Because the design map used inertial velocity magnitude and flight path angle, extra care had to be taken to compare to the MRV, which had an initial condition with a different latitude and azimuth. It may be preferable to use relative velocity magnitude and flight path angle for future design maps. 


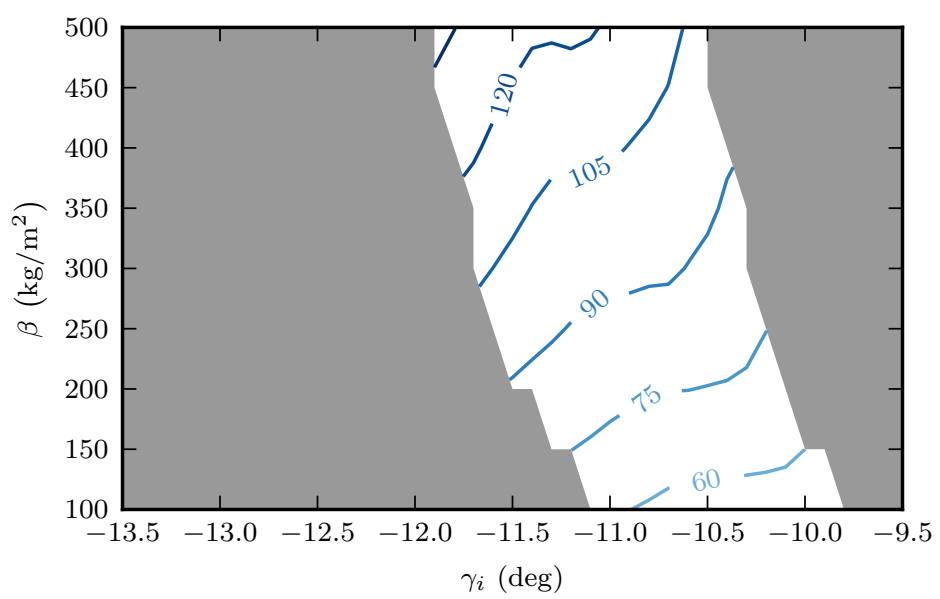

(a) Peak heat rate $\left(\mathrm{W} / \mathrm{cm}^{2}\right)$ as a function of $\gamma_{i}$ and $\beta$ with $L / D$ and $V_{i}$ fixed

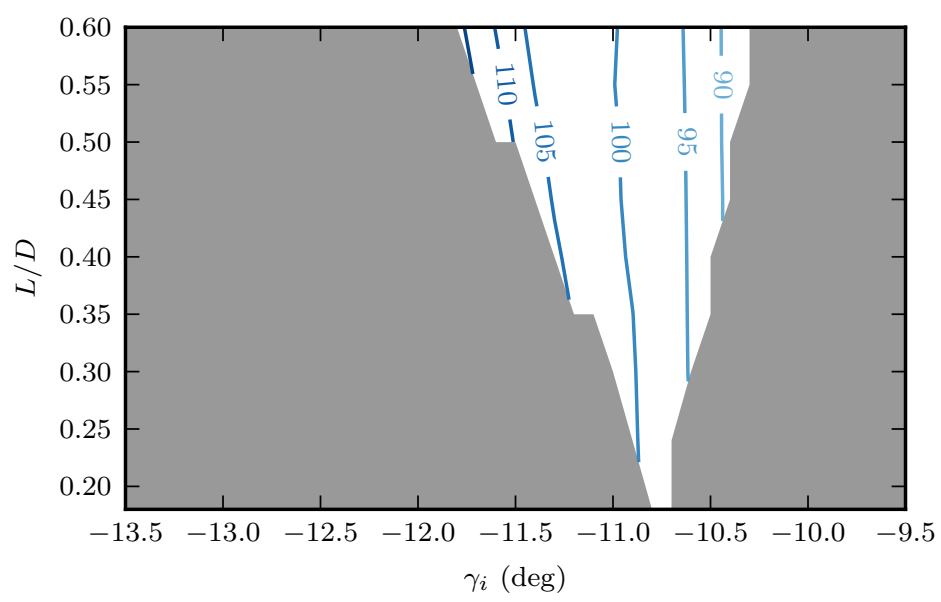

(b) Peak heat rate $\left(\mathrm{W} / \mathrm{cm}^{2}\right)$ as a function of $\gamma_{i}$ and $L / D$ with $\beta$ and $V_{i}$ fixed

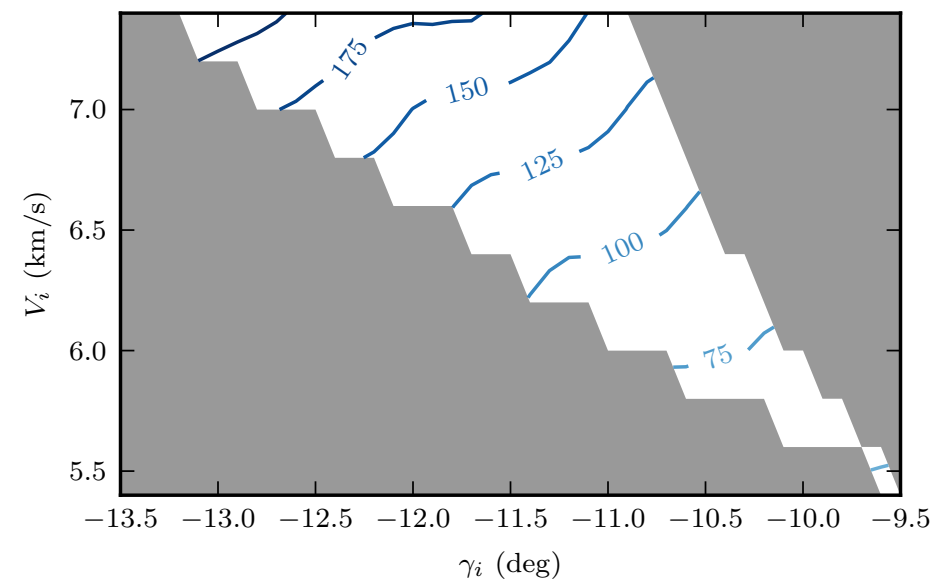

(c) Peak heat rate $\left(\mathrm{W} / \mathrm{cm}^{2}\right)$ as a function of $\gamma_{i}$ and $V_{i}$ with $\beta$ and $L / D$ fixed

Figure 11: Peak heat rate sensitivity to vehicle design parameters 


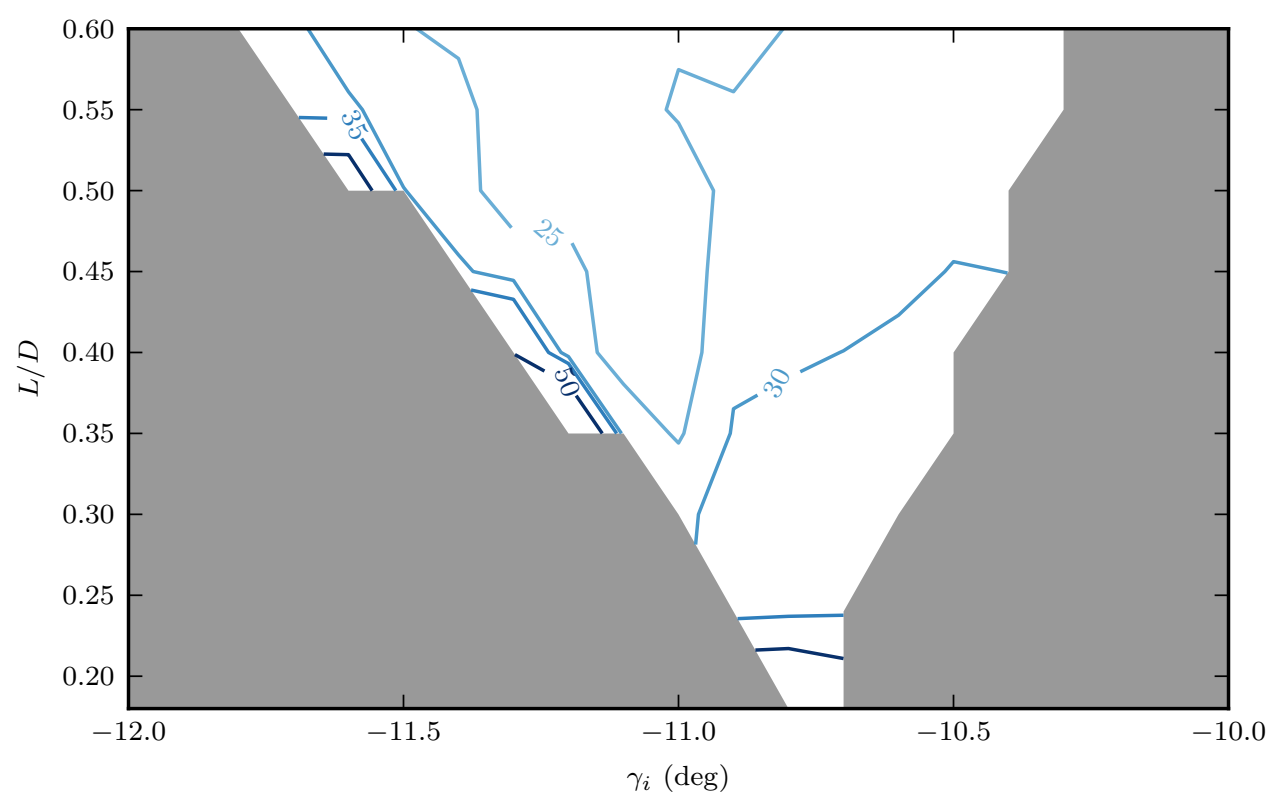

Figure 12: In-plane $\Delta V$ for a fixed $\beta$

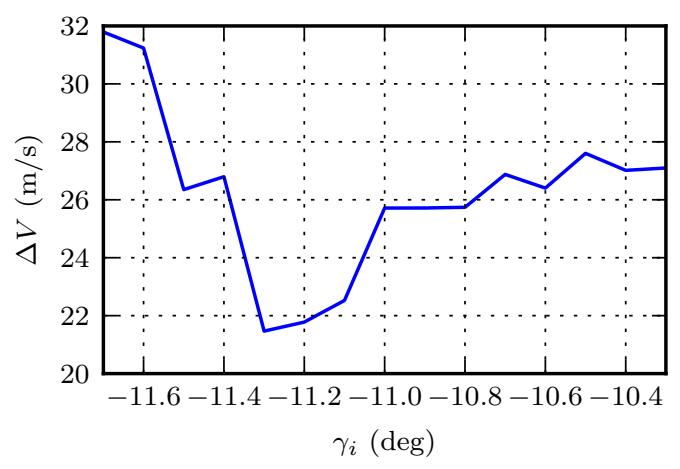

(a) $\Delta V(\mathrm{~m} / \mathrm{s})$

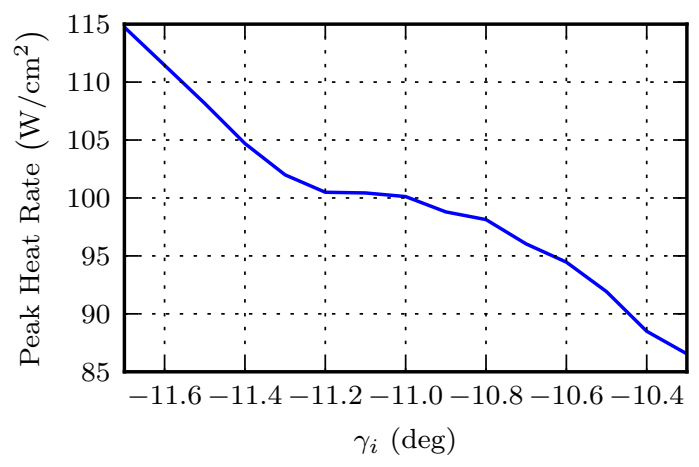

(c) Peak heat rate $\left(\mathrm{W} / \mathrm{cm}^{2}\right)$

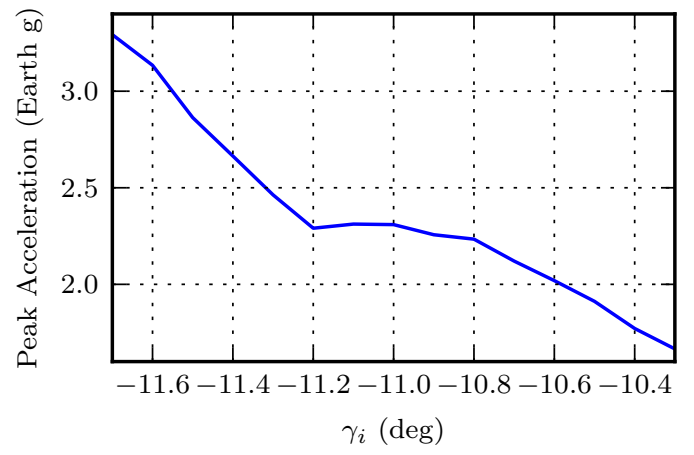

(b) Peak sensed acceleration magnitude (Earth g)

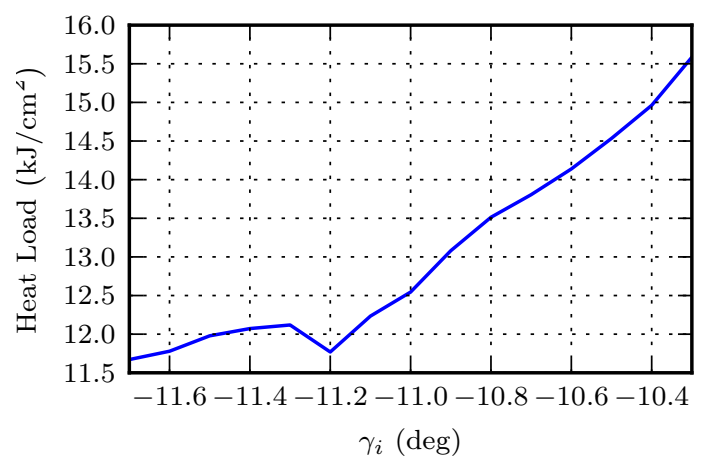

(d) Heat load $\left(\mathrm{kJ} / \mathrm{cm}^{2}\right)$

Figure 13: For a fixed $\beta$ and $L / D, \gamma_{i}$ can be selected 


\section{Conclusion}

Two studies demonstrated the capabilities of FNPAG for Mars aerocapture maneuvers. First, a Monte Carlo analysis of the MRV design showed that FNPAG is able to guide the human scale vehicle while requiring a post-aerocapture in-plane orbital correction $\Delta V$ of $40 \mathrm{~m} / \mathrm{s}$. Second, a design map covering a large range of ballistic coefficients, lift-to-drag ratios, and atmospheric entry conditions showed the adaptability of FNPAG without the need to generate reference trajectories or tune guidance parameters. The design map also showed that FNPAG has stable performance across a wide range of vehicle parameters and trajectory conditions. Lastly, the utility of the design map was illustrated through a variety of sample applications, using the MRV design as an example.

\section{References}

${ }^{1}$ Lu, P., Cerimele, C. J., Tigges, M. A., and Matz, D. A., "Optimal Aerocapture Guidance,” Journal of Guidance, Control, and Dynamics, Vol. 38, No. 4, 2015, pp. 553-565. doi:10.2514/1.G000713

${ }^{2}$ Cerimele, C. J., Robertson, E. A., Sostaric, R. R., Campbell, C. H., Robinson, P. E., Hershey, M. P., Stachowiak, S. J., Matz, D. A., Braden, E. M., Johnson, B. J., Garcia, J. A., Bowles, J. V., Kinney, D. J., Theisinger, J. E., "A Rigid Mid Liftto-Drag Ratio Approach to Human Mars Entry, Descent, and Landing," AIAA Guidance, Navigation, and Control Conference, Jan. 2017.

${ }^{3}$ Breakwell, J. V., Gillespie, R. W., and Ross, S., "Researches in Interplanetary Transfer," ARS Journal, Vol. 31, No. 2, 1961, pp. 201-208. doi:10.2514/8.5428

${ }^{4}$ Mendeck, G. F., and Craig, L. E., "Entry Guidance for the 2011 Mars Science Laboratory Mission," AIAA Atmospheric Flight Mechanics Conference, Aug. 2011. doi:10.2514/6.2011-6639

${ }^{5}$ Smith, K. M., "Predictive Lateral Logic for Numerical Entry Guidance Algorithms", 26th AAS/AIAA Space Flight Mechanics Meeting, Feb. 2016.

${ }^{6}$ Justh, H. L., "Mars Global Reference Atmospheric Model 2010 Version: Users Guide," NASA/TM-2014-217499, Feb. 2014.

${ }^{7}$ Drake, B. G., "Human Exploration of Mars: Design Reference Architecture 5.0," NASA/SP-2009-566, Jul. 2009.

8 "Entry, Descent, and Landing Systems Analysis Study: Phase 1 Report," NASA/TM-2010-216720, Jul. 2010. 